\title{
Teste de condutividade elétrica para sementes de mamoneira
}

\author{
Electrical conductivity test on castor bean seeds
}

\author{
Líbia Belisário da Silva ${ }^{1}$; Cibele Chalita Martins ${ }^{2}$
}

\section{Resumo}

Com o objetivo de verificar a eficiência do teste de condutividade elétrica na avaliação da emergência de plântulas em campo de sementes de mamoneira, cinco lotes da cv. AL Guarany 2002 foram submetidos aos seguintes testes: germinação, primeira contagem de germinação, envelhecimento acelerado $\left(45^{\circ} \mathrm{C}\right.$ por $24 \mathrm{~h}$ a $100 \% \mathrm{UR}$ ), emergência de plântulas em campo, índice de velocidade de emergência de plântulas e condutividade elétrica, testando-se os períodos de condicionamento (2, 4, 6, 8 e 24 horas) e o número de sementes $(25,50$ e 75$)$ em $75 \mathrm{ml}$ de água destilada a $25^{\circ} \mathrm{C}$. Empregou-se delineamento inteiramente casualizado. As médias foram comparadas pelo teste de Tukey a $5 \%$ de probabilidade. Correlacionaramse os dados médios dos testes realizados em laboratório e em campo. O teste de condutividade elétrica por períodos de embebição de 4 horas com 25 sementes e 6 horas com 50 sementes e o índice de velocidade de emergência mostraram-se eficientes na seleção de lotes quanto ao vigor fornecendo informação equivalente à emergência de plântulas em campo.

Palavras-chave: Mamona, Ricinus communis L, velocidade de emergência, vigor

\begin{abstract}
The aim was to study the efficiency of electrical conductivity test in evaluating the quality of castor seeds. Emergence of seedlings was evaluated in the field, with five seed lots of cv. AL Guarany 2002. The seeds were submitted to the following tests: germination; first count of germination; accelerated aging $\left(45^{\circ} \mathrm{C}\right.$ by 24 to $100 \%$ UR), emergence of seedlings in the field, rate of speed of development of seedlings and electrical conductivity, testing the periods (2, 4, 6, 8 and 24 hours) and the number of seeds $(25,50$ and 75$)$ in $75 \mathrm{ml}$ of distilled water conditioning to $25^{\circ} \mathrm{C}$. A completely randomized design was used. The averages were compared by the Tukey test at $5 \%$. The mean laboratory and field test data were correlated. Electrical conductivity test for 4 hours with 25 seeds and 6 hours with 50 seeds, and the rate of speed emergency proved efficient in the selection of lots vigor by providing information equivalent to the emergence of seedlings in the field.
\end{abstract}

Key words: Castor bean, Ricinus communis L, speed emergency, vigor

${ }^{1}$ Eng ${ }^{\mathrm{a}}$. Agr ${ }^{\mathrm{a}}$. Mestranda em Produção e Tecnologia de Sementes - FCA/UNESP; E-mail: libiabel@yahoo.com.br

2 Prof $^{a}$ Dr $^{\mathrm{a}}$ Depto. de Agricultura/Melhoramento Vegetal, FCA/UNESP.

* Autor para correspondência 


\section{Introdução}

A mamona se destaca no Brasil como uma das matérias primas para a produção de biodiesel. No entanto, o seu cultivo ainda é realizado com sementes dos próprios produtores e um dos maiores entraves para a expansão da cultura no país referese, principalmente, a escassez e baixa qualidade das sementes utilizadas (FORNAZIERI JÚNIOR, 1986). Dentre os métodos disponíveis para avaliar a qualidade das sementes, apenas o teste de germinação tem sido utilizado para a mamona (BRASIL, 1992).

No entanto, as empresas produtoras de sementes de diversas espécies agrícolas e as instituições oficiais têm incluído os testes de vigor em programas internos de controle de qualidade para a determinação da qualidade fisiológica das sementes (MARCOS FILHO, 1999). Freqüentemente, lotes que apresentam germinação semelhante exibem comportamentos distintos no campo ou armazenamento. Essas diferenças podem ser atribuídas ao fato das primeiras alterações nos processos bioquímicos associados à deterioração ocorrerem, geralmente, antes que sejam verificados declínios na capacidade germinativa (DELOUCHE; BASKIN, 1973).

Considerando-se que os testes de vigor fornecem índices mais sensíveis do potencial fisiológico, quando comparados ao teste de germinação (ASSOCIATION OF OFFICIAL SEED ANALYSTIS, 1983), qualquer evento que preceda a perda do poder germinativo pode servir como base para o desenvolvimento de testes de vigor. Entretanto, acredita-se que, quanto mais distante da perda do poder germinativo estiver a variável avaliada, mais sensível deverá ser o teste. Como a degradação das membranas celulares se constitui num dos eventos iniciais do processo de deterioração (DELOUCHE; BASKIN, 1973), os testes que avaliam a integridade das membranas, como o de condutividade elétrica, seriam, teoricamente, os mais sensíveis para estimar o vigor.
No teste de condutividade elétrica, a qualidade das sementes é avaliada indiretamente através da determinação da quantidade de lixiviados na solução de embebição das sementes. Os menores valores, correspondentes à menor liberação de exsudatos, indicam alto potencial fisiológico (maior vigor), revelando menor intensidade de desorganização dos sistemas membranais das células (VIEIRA; KRZYZANOWSKI, 1999).

No entanto, vários fatores podem afetar os resultados do teste de condutividade elétrica, como por exemplo: o tempo de embebição, o tamanho da semente, a temperatura de embebição, o teor de água inicial das sementes, o número de sementes da amostra e o genótipo (DIAS; MARCOS FILHO, 1995; MENEZES; PASINATTO, 1995; VIEIRA et al., 1996; BRANDÃO JÚNIOR et al., 1997; RIBEIRO; CARVALHO; SALGADO， 1997; RODO et al., 1998; DIAS; VIEIRA; BHÉRING, 1998; VANZOLINI, 1998; SÁ, 1999; VIEIRA; KRZYZANOWSKI, 1999; MARTINS et. al., 2002; GASPAR; NAKAGAWA, 2002; DUTRA; VIEIRA, 2006) e isso tem levado tecnologistas e pesquisadores a estudarem melhor a sua metodologia para as diferentes espécies.

A metodologia convencional de condutividade elétrica recomenda um período de 24 horas de embebição. Porém, alguns trabalhos de pesquisa mostram que esse período pode ser menor para algumas espécies. Resultados eficientes para a distinção de lotes de sementes de soja e feijão vagem quanto ao vigor foram obtidos em um período máximo de 20 horas de embebição(DIAS; MARCOS FILHO, 1995; DIAS; VIEIRA; BHÉRING, 1998), para girassol em 18 horas (BRANDÃO JÚNIOR et al., 1997), para milho em 12 horas (RIBEIRO; CARVALHO; SALGADO, 1997), para couvebrócolos e abobrinha em oito horas (MARTINS et. al., 2002; DUTRA; VIEIRA, 2006), para tomate em seis horas (SÁ, 1999), para quiabo em quatro horas (DIAS; VIEIRA; BHÉRING, 1998), para amendoim em três horas (VANZOLINI, 1998) e para milheto em duas horas (GASPAR; NAKAGAWA, 2002). 
Testes de vigor que avaliem a qualidade fisiológica das sementes rapidamente encurtam o período de tomada de decisão da indústria (MARCOS FILHO et al., 1990; DIAS; MARCOS FILHO, 1995).

Quanto ao número de sementes da amostra necessário para a condução do teste de condutividade elétrica existe a recomendação de quatro repetições de 50 sementes como forma de reduzir o coeficiente de variação (LOEFFLER; TEKRONY; EGLI, 1988; VIEIRA; KRZYZANOWSKI, 1999). Alguns trabalhos, visando adequar o teste a cada espécie, avaliaram diferentes números de sementes. Para milho e tomate da cultivar Kada repetições de 25 sementes apresentaram melhores resultados do que 50 sementes (RIBEIRO; CARVALHO; SALGADO, 1997; RODO et al., 1998); no entanto, para tomate da cultivar IAC, repetições de 50 sementes mostraram maior eficiência (RODO et al., 1998). Sá (1999) constatou que a utilização de amostras entre 25 e 100 sementes não afeta os valores de condutividade elétrica das cultivares de tomate Petomech e Santa Clara. Para sementes de milheto, aveia preta e azevém melhores resultados são obtidos com amostras de 25 sementes (MENEZES; PASINATTO, 1995).

Assim, este trabalho tem como objetivo verificar a eficiência do teste de condutividade elétrica na avaliação da qualidade de lotes de sementes de mamoneira visando à emergência de plântulas em campo.

\section{Material e métodos}

Esta pesquisa foi realizada no Laboratório de Análise de Sementes do Departamento de Produção Vegetal da Faculdade de Ciências Agronômicas da UNESP, submetendo-se cinco lotes de sementes de mamona (Ricinus communis L.), cultivar AL Guarany 2002 às seguintes determinações e testes de avaliação de qualidade: Teor de água das sementes - determinado antes e após os testes de envelhecimento acelerado e de condutividade elétrica, utilizando-se quatro subamostras de 10 sementes para cada lote, adotando-se o método da estufa a $105 \pm 3^{\circ} \mathrm{C} / 24 \mathrm{~h}$ (BRASIL, 1992). Germinação - foi conduzida com oito subamostras de 25 sementes por repetição, em rolo de papel toalha (RP), previamente umedecido com água destilada na proporção de 2,5 vezes o peso do substrato, à temperatura alternada de $20-30^{\circ} \mathrm{C}$, determinandose a porcentagem de plântulas normais, avaliadas no sétimo e décimo quarto dias após a instalação (BRASIL, 1992). Primeira contagem da germinação - foi avaliada considerando-se as plântulas normais presentes no sétimo dia do teste de germinação (BRASIL, 1992). Envelhecimento acelerado - foi conduzido com uma camada única de sementes de mamona sobre tela em caixa plástica contendo $40 \mathrm{~mL}$ de água, mantidas a $45^{\circ} \mathrm{C}$ por 24 horas, seguindo-se da determinação do vigor das sementes utilizando o mesmo procedimento do teste de germinação, com umedecimento de duas vezes o peso do papel em água e contagem no sétimo dia (HAMPTON; TEKRONY, 1995; SOUZA et al., 2007). Emergência de plântulas em campo - utilizando-se quatro subamostras de 100 sementes por lote, semeadas a cinco $\mathrm{cm}$ de profundidade no espaçamento de $40 \mathrm{~cm}$ entre linhas, contabilizando-se as plântulas emersas aos 21 dias após a semeadura. Índice de velocidade de emergência de plântula - foi conduzido em conjunto com o teste de emergência em campo, contabilizando-se diariamente, no mesmo horário, o número de plântulas normais emersas, seguindo a metodologia proposta por Maguire (1962). Condutividade Elétrica - as sementes foram pesadas com precisão de quatro casas decimais e colocadas para embeber em copos plásticos descartáveis (capacidade de $200 \mathrm{ml}$ ), contendo 75 $\mathrm{ml}$ de água destilada (condutividade elétrica $\leq 2$ $\left.\mu \mathrm{S} . \mathrm{cm}^{-1}\right)$ na temperatura de $25^{\circ} \mathrm{C}$. Foram testadas repetições de 25,50 e 75 sementes e as leituras foram efetuadas após 2, 4, 6, 8 e 24 horas, para que o teste pudesse adequar-se ao horário de expediente de um laboratório de rotina de análise de sementes. Após cada período de embebição, a condutividade elétrica da solução foi determinada por meio de 
leituras em condutivímetro DIGIMED, modelo DM 31, com os resultados expressos em $\mathrm{mS} . \mathrm{cm}^{-1} \cdot \mathrm{g}^{-1} \mathrm{de}$ semente. À exceção dos fatores estudados, o teste foi conduzido conforme descrito por Hampton e TeKrony (1995) e Vieira e Krzyzanowski (1999).

No procedimento estatístico, a análise de variância foi realizada separadamente para cada teste e, conjuntamente, mediante análise de correlação com a emergência em campo, segundo delineamento inteiramente casualizado, onde a comparação entre as médias dos lotes foi efetuada pelo teste de Tukey, ao nível de $5 \%$ de probabilidade. As porcentagens, se necessário, foram previamente transformadas para posteriormente serem submetidos à análise (GOMES, 2000).

\section{Resultados e discussão}

Os teores de água iniciais das sementes foram semelhantes para os cinco lotes (Tabela 1), situandose entre 12,5 e 13,5\%, ou seja, com variação máxima de um ponto porcentual, inferior à amplitude máxima de 1 a 2 pontos percentuais, aceita para garantir a credibilidade dos resultados obtidos nos testes de vigor (MARCOS FILHO, 1999). Embora na literatura não tenham sido encontrados valores de teor de água de referência para sementes de mamoneira, os teores de água iniciais situaram-se dentro dos limites recomendados para a avaliação da condutividade elétrica de soja e feijão mungo, que estaria entre $10 \%$ e $17 \%$ (AOSA, 1983; LOEFFLER; TEKRONY; EGLI, 1988; HAMPTON; JOHNSTONE; EUAUMPON, 1992; VIEIRA et al., 2002).

Tabela 1. Teores de água inicial, após o envelhecimento acelerado e após a imersão das sementes em água para o teste de condutividade elétrica (CE) nos tempos de 2, 4, 6, 8 e 24 horas em repetições de 25, 50 e 75 sementes de cinco lotes de mamona. Botucatu-SP, 2006.

\begin{tabular}{|c|c|c|c|c|c|c|}
\hline \multirow{2}{*}{$\begin{array}{c}\text { Teor de água } \\
\text { das sementes }(\%)\end{array}$} & \multicolumn{5}{|c|}{ Lotes } & \multirow{2}{*}{$\begin{array}{l}\mathrm{CV} \\
(\%)\end{array}$} \\
\hline & 1 & 2 & 3 & 4 & 5 & \\
\hline Inicial & $13,0 \mathrm{ab}$ & $13,5 \mathrm{a}$ & $12,5 \mathrm{~b}$ & $13,2 \mathrm{ab}$ & $13,4 \mathrm{a}$ & 3,12 \\
\hline Envelhecimento acelerado & $20,3 \mathrm{a}$ & $20,4 \mathrm{a}$ & $20,6 \mathrm{a}$ & $21,0 \mathrm{a}$ & $20,4 \mathrm{a}$ & 3,81 \\
\hline CE 2 h 25 sementes & $16,6 \mathrm{a}$ & $16,6 \mathrm{a}$ & $16,5 \mathrm{a}$ & $16,6 \mathrm{a}$ & $16,1 \mathrm{a}$ & 2,93 \\
\hline 50 sementes & $15,0 \mathrm{~b}$ & $17,8 \mathrm{a}$ & $16,5 \mathrm{a}$ & $15,4 \mathrm{ab}$ & $17,1 \mathrm{a}$ & 3,41 \\
\hline 75 sementes & $16,7 \mathrm{ab}$ & $17,6 \mathrm{a}$ & $16,9 \mathrm{a}$ & $15,6 \mathrm{~b}$ & $17,8 \mathrm{a}$ & 3,16 \\
\hline CE 4 h 25 sementes & $16,2 \mathrm{~b}$ & $19,4 \mathrm{a}$ & $17,2 \mathrm{ab}$ & $18,7 \mathrm{a}$ & $19,9 \mathrm{a}$ & 3,63 \\
\hline 50 sementes & $18,0 \mathrm{ab}$ & $21,0 \mathrm{a}$ & $17,3 \mathrm{~b}$ & $19,3 \mathrm{a}$ & $19,5 \mathrm{a}$ & 3,54 \\
\hline 75 sementes & $17,2 \mathrm{~b}$ & $20,1 \mathrm{a}$ & $17,6 \mathrm{~b}$ & $19,4 \mathrm{ab}$ & $20,6 \mathrm{a}$ & 3,17 \\
\hline CE 6 h 25 sementes & $20,9 \mathrm{a}$ & $20,5 \mathrm{a}$ & $19,4 \mathrm{a}$ & $20,6 \mathrm{a}$ & 19,9 a & 2,96 \\
\hline 50 sementes & $20,3 \mathrm{a}$ & $21,8 \mathrm{a}$ & $19,0 \mathrm{~b}$ & $20,5 \mathrm{a}$ & $20,0 \mathrm{a}$ & 3,35 \\
\hline 75 sementes & $22,0 \mathrm{a}$ & $20,9 \mathrm{a}$ & $19,2 \mathrm{a}$ & $20,3 \mathrm{a}$ & $20,9 \mathrm{a}$ & 3,93 \\
\hline CE 8 h 25 sementes & $21,5 \mathrm{a}$ & $22,6 \mathrm{a}$ & $21,6 \mathrm{a}$ & $21,1 \mathrm{a}$ & $22,7 \mathrm{a}$ & 3,24 \\
\hline 50 sementes & $19,8 \mathrm{~b}$ & $25,0 \mathrm{a}$ & $21,4 \mathrm{~b}$ & $21,1 \mathrm{~b}$ & $22,5 \mathrm{ab}$ & 3,71 \\
\hline 75 sementes & $22,3 \mathrm{ab}$ & $25,3 \mathrm{a}$ & $21,1 \mathrm{~b}$ & $20,8 \mathrm{~b}$ & $23,0 \mathrm{ab}$ & 2,85 \\
\hline CE 24 h 25 sementes & $30,3 \mathrm{a}$ & $31,2 \mathrm{a}$ & $26,1 \mathrm{~b}$ & $29,5 \mathrm{a}$ & $31,7 \mathrm{a}$ & 3,44 \\
\hline 50 sementes & $28,5 \mathrm{~b}$ & $26,8 \mathrm{~b}$ & $28,2 \mathrm{~b}$ & $26,7 \mathrm{~b}$ & $32,8 \mathrm{a}$ & 2,93 \\
\hline 75 sementes & $27,4 \mathrm{c}$ & $37,7 \mathrm{a}$ & $27,0 \mathrm{~cd}$ & $24,4 \mathrm{~d}$ & $33,5 \mathrm{~b}$ & 3,35 \\
\hline
\end{tabular}

${ }^{1}$ Médias seguidas pela mesma letra, na linha, não diferem entre si pelo teste de Tukey, em nível de 5\% de probabilidade. 
O teor de água obtido ao final do teste de envelhecimento acelerado é um indicador da uniformidade das condições do teste e a variação máxima verificada entre os lotes foi de $0,7 \%$, inferior à tolerada, entre 3 e 4\% (MARCOS FILHO, 1999).

Observou-se, também, no teste de condutividade um aumento do teor de água das sementes com o aumento do período de embebição, que confirma resultados de pesquisas sobre o assunto (LOEFFLER; TEKRONY; EGLI, 1988; WANG; HAMPTON; HILL, 1994; VIEIRA et al., 1996; DIAS; MARCOS FILHO, 1996; DIAS; VIEIRA; BHÉRING, 1998; VANZOLINI, 1998; DUTRA; VIEIRA, 2006).
Na Tabela 2, observa-se que os únicos testes que apresentaram resultados correlacionados com a emergência de plântulas em campo, que foi usada como referência, foram o índice de velocidade de emergência e o teste de condutividade elétrica com período de embebição e subamostras de, respectivamente, 4 horas e 25 sementes ou 6 horas e 50 sementes. Embora as correlações entre esses últimos testes e a emergência de plântulas em campo tenham sido baixas, foram estatisticamente significativas e os resultados obtidos, quanto à classificação do vigor dos lotes, assemelharam-se aos obtidos na maioria dos demais testes de vigor.

Tabela 2. Coeficiente de correlação simples (r) entre os resultados dos testes de laboratório e da emergência de plântulas em campo para as sementes de cinco lotes de mamona. Botucatu-SP, 2006.

\begin{tabular}{|c|c|}
\hline Testes de laboratório x Emergência em campo & $\mathbf{R}$ \\
\hline Germinação & $0,313^{\text {ns }}$ \\
\hline Primeira contagem & $0,067^{\mathrm{ns}}$ \\
\hline Envelhecimento acelerado & $0,120^{\text {ns }}$ \\
\hline IVE & $0,893 * * *$ \\
\hline \multicolumn{2}{|l|}{ Condutividade elétrica } \\
\hline $2 \mathrm{~h} 25$ sementes & $-0,344^{\mathrm{ns}}$ \\
\hline 50 sementes & $-0,358^{\mathrm{ns}}$ \\
\hline 75 sementes & $-0,409^{\mathrm{ns}}$ \\
\hline $4 \mathrm{~h} 25$ sementes & $-0,483^{*}$ \\
\hline 50 sementes & $-0,226^{\mathrm{ns}}$ \\
\hline 75 sementes & $-0,157^{\mathrm{ns}}$ \\
\hline $6 \mathrm{~h} 25$ sementes & $-0,248^{\text {ns }}$ \\
\hline 50 sementes & $-0,458^{*}$ \\
\hline 75 sementes & $-0,388^{\text {ns }}$ \\
\hline $8 \mathrm{~h} 25$ sementes & $-0,344^{\mathrm{ns}}$ \\
\hline 50 sementes & $-0,155^{\text {ns }}$ \\
\hline 75 sementes & $-0,075^{\mathrm{ns}}$ \\
\hline $24 \mathrm{~h} 25$ sementes & $-0,433^{\text {ns }}$ \\
\hline 50 sementes & $-0,322^{\mathrm{ns}}$ \\
\hline 75 sementes & $-0,321^{\text {ns }}$ \\
\hline
\end{tabular}

Não significativo (ns), significativo a $5 \%\left(^{*}\right)$ e a $0,1(* * *)$ de probabilidade. 
Os dados obtidos na condutividade elétrica apresentaram correlação negativa com a emergência de plântulas em campo, mostrando que aumentos nos valores de condutividade elétrica corresponderam à reduções na porcentagem de emergência; tais resultados estão de acordo com aqueles verificados na literatura (POWELL, 1986; MARCOS FILHO et al., 1990).

Para uma análise mais adequada da eficiência dos testes, também foi realizada a comparação das médias dos lotes para cada parâmetro avaliado
(Tabela 3). Deve-se destacar que, com exceção do lote 5, os lotes apresentavam germinação superior ao mínimo estabelecido pelos Padrões de Sementes para a comercialização para mamoneira (BRASIL, 2005) e em valores estatisticamente semelhantes. Essas características são importantes para a confiabilidade da aplicação dos resultados do trabalho, pois os testes de vigor devem ser capazes de detectar diferenças no potencial fisiológico de lotes, principalmente dos que possuem poder germinativo semelhante (TORRES; MARCOS FILHO, 2001).

Tabela 3. Testes de germinação e de vigor avaliado pela primeira contagem de germinação, envelhecimento acelerado, emergência de plântulas em campo, índice de velocidade de emergência (IVE) e condutividade elétrica (CE) nos tempos de 2, 4, 6, 8 e 24 horas com 25, 50 e 75 sementes de cinco lotes de mamona. Botucatu-SP, 2006.

\begin{tabular}{|c|c|c|c|c|c|c|}
\hline \multirow[t]{2}{*}{ Qualidade das sementes } & \multicolumn{5}{|c|}{ Lotes } & \multirow{2}{*}{$\begin{array}{l}\text { C.V } \\
(\%)\end{array}$} \\
\hline & 1 & 2 & 3 & 4 & 5 & \\
\hline Germinação (\%) & $88,1 \mathrm{a}$ & $88,5 \mathrm{a}$ & 84,6 a & $80,2 \mathrm{a}$ & $62,6 \mathrm{~b}$ & 16,0 \\
\hline Primeira contagem $(\%)$ & $38,8 \mathrm{abc}$ & $43,2 \mathrm{ab}$ & $32,9 \mathrm{bc}$ & $46,1 \mathrm{a}$ & $29,1 \mathrm{c}$ & 18,9 \\
\hline Envelhecimento acelerado (\%) & $16,3 \mathrm{~b}$ & $47,3 \mathrm{a}$ & $60,1 \mathrm{a}$ & $51,1 \mathrm{a}$ & $45,2 \mathrm{a}$ & 28,4 \\
\hline Emergência de plântulas (\%) & $57,5 \mathrm{a}$ & $50,2 \mathrm{ab}$ & $52,6 \mathrm{ab}$ & 48,4 bc & $42,1 \mathrm{c}$ & 7,3 \\
\hline IVE & $8,5 \mathrm{a}$ & $5,4 \mathrm{bc}$ & $6,2 \mathrm{~b}$ & $5,6 \mathrm{bc}$ & $4,1 \mathrm{c}$ & 13,9 \\
\hline \multicolumn{7}{|l|}{$\mathrm{CE}\left(\mu \mathrm{S} \cdot \mathrm{cm}^{-1} \cdot \mathrm{g}^{-1}\right)$} \\
\hline $2 \mathrm{~h} 25$ sementes & $30,95 \mathrm{~b}$ & $37,34 \mathrm{ab}$ & $37,34 \mathrm{ab}$ & $32,05 \mathrm{~b}$ & $43,01 \mathrm{a}$ & 4,6 \\
\hline 50 sementes & $26,80 \mathrm{c}$ & $32,16 \mathrm{bc}$ & 34,94 ba & $30,60 \mathrm{bc}$ & $39,45 \mathrm{a}$ & 5,1 \\
\hline 75 sementes & $25,80 \mathrm{~b}$ & $30,94 \mathrm{ab}$ & $32,18 \mathrm{ab}$ & $28,88 \mathrm{~b}$ & $36,70 \mathrm{a}$ & 5,4 \\
\hline $4 \mathrm{~h} 25$ sementes & $31,29 \mathrm{c}$ & $41,91 \mathrm{~b}$ & $40,79 \mathrm{~b}$ & $39,66 \mathrm{~b}$ & $48,59 \mathrm{a}$ & 4,2 \\
\hline 50 sementes & $29,35 \mathrm{c}$ & $41,55 \mathrm{a}$ & $40,77 \mathrm{ab}$ & $34,98 \mathrm{cb}$ & $43,40 \mathrm{a}$ & 4,4 \\
\hline 75 sementes & $26,44 \mathrm{c}$ & $38,01 \mathrm{ab}$ & $39,26 \mathrm{a}$ & $32,67 \mathrm{bc}$ & $42,14 \mathrm{a}$ & 4,7 \\
\hline $6 \mathrm{~h} \quad 25$ sementes & $37,38 \mathrm{c}$ & $42,59 \mathrm{ab}$ & $46,24 \mathrm{a}$ & $42,81 \mathrm{ab}$ & 48,64 a & 3,9 \\
\hline 50 sementes & $32,40 \mathrm{~d}$ & $44,57 \mathrm{~b}$ & $43,40 \mathrm{bc}$ & $37,90 \mathrm{dc}$ & $55,33 \mathrm{a}$ & 3,9 \\
\hline 75 sementes & $33,80 \mathrm{c}$ & $39,98 \mathrm{abc}$ & $41,55 \mathrm{ab}$ & $38,62 \mathrm{bc}$ & $45,89 \mathrm{a}$ & 4,2 \\
\hline $8 \mathrm{~h} \quad 25$ sementes & $38,93 \mathrm{c}$ & $43,69 \mathrm{bc}$ & $48,75 \mathrm{ab}$ & $42,83 \mathrm{bc}$ & 54,98 a & 3,7 \\
\hline 50 sementes & $36,65 \mathrm{~b}$ & $46,94 \mathrm{a}$ & $47,25 \mathrm{a}$ & 39,12 b & $47,51 \mathrm{a}$ & 3,9 \\
\hline 75 sementes & $34,77 \mathrm{c}$ & $45,74 \mathrm{ab}$ & $46,52 \mathrm{a}$ & $39,23 \mathrm{bc}$ & $45,23 \mathrm{ab}$ & 4,0 \\
\hline 24 h 25 sementes & $57,54 \mathrm{~d}$ & $68,72 \mathrm{bc}$ & $72,18 \mathrm{~b}$ & $65,29 \mathrm{c}$ & 88,03 a & 2,4 \\
\hline 50 sementes & $54,12 \mathrm{~d}$ & $71,38 \mathrm{~b}$ & $69,00 \mathrm{bc}$ & $63,11 \mathrm{c}$ & $78,04 \mathrm{a}$ & 2,5 \\
\hline 75 sementes & $53,71 \mathrm{c}$ & $68,82 \mathrm{a}$ & $65,52 \mathrm{ab}$ & $62,19 \mathrm{~b}$ & $70,00 \mathrm{a}$ & 2,6 \\
\hline
\end{tabular}

*Médias seguidas pela mesma letra, na linha, não diferem entre si pelo teste de Tukey, em nível de 1\% de probabilidade. 
Verificou-se que os resultados do teste de emergência de plântulas em campo, índice de velocidade de emergência e condutividade elétrica nas combinações de 4 horas com 25 sementes e 6 horas com 50 sementes foram capazes de selecionar o lote de maior e de menor vigor, que foram os lotes 1 e 5, respectivamente. Esses resultados assemelham-se aos obtidos na maioria dos demais testes de vigor, exceto no teste da primeira contagem e do envelhecimento acelerado, que apresentaram resultados discordantes e, por isso, menos confiáveis.

Os demais lotes, de qualidade fisiológica intermediária, foram selecionados em classes de vigor que dependeram da seletividade do teste utilizado. Assim, verificou-se que o teste de emergência de plântulas em campo separou os lotes em quatro classes de vigor: alto (lote 1), médioalto (2 e 3), médio-baixo (lote 4) e baixo (lote 5). O índice de velocidade de emergência também separou os lotes em quatro classes de vigor: alto (lote 1), médio (lote 3 ), médio-baixo (lote 2 e 4 ) e baixo (lote 5). O teste de condutividade elétrica avaliado após embebição por 4 horas com subamostras de 25 sementes separou os lotes em três classes de vigor, alto (lote 1), médio (lotes 2, 3 e 4) e baixo (lote 5); o de condutividade elétrica após 6 horas com 25 sementes, separou os lotes em alto (lote 1), médioalto (lote 4), médio (lote 3 ), médio-baixo (lote 2) e baixo vigor (lote 5 ).

Essas metodologias propostas para o teste de condutividade elétrica apresentam grande vantagem em comparação ao índice de velocidade de emergência (IVE), pela facilidade de execução e rapidez na obtenção dos resultados, embora tenham apresentado menor correlação com a emergência em campo que o IVE (Tabela 2). Assim, a avaliação de vigor pelo teste de condutividade elétrica mostra-se promissor para sementes de mamoneira, indicando a necessidade da continuidade desses estudos.

\section{Conclusão}

O teste de condutividade elétrica por períodos de embebição de 4 horas com 25 sementes e 6 horas com 50 sementes e o índice de velocidade de emergência mostraram-se eficientes na seleção de lotes quanto ao vigor fornecendo informação equivalente à emergência de plântulas em campo.

\section{Referências}

ASSOCIATION OF OFFICIAL SEED ANALYSTIS AOSA. Seed vigour testing handbook. Lincoln: AOSA, 1983. (Contribuition, 32).

BRANDÃO JUNIOR, D. S.; RIBEIRO, D. C. A.; BERNADINO FILHO, J. R.; VIEIRA, M. C. C. C. Adequação do teste de condutividade elétrica para determinar a qualidade fisiológica de sementes de girassol. Informativo Abrates, Londrina, v. 7, n. 1/2, p. 182-184, 1997.

BRASIL. Ministério da Agricultura e Reforma Agrária. Secretaria Nacional de Defesa Agropecuária. Departamento Nacional de Defesa Vegetal. Coordenação de Laboratório Vegetal. Regras para análise de sementes. Brasília, 1992.

BRASIL. Ministério da Agricultura, Pecuária e Abastecimento. Padrões para produção e comercialização de sementes de mamona Cultivares não híbridas (Variedades). Instrução normativa $\mathrm{n}^{\circ} 25$, de 16 de dezembro de 2005. Diário Oficial da União, Brasília, sec.1, n. 243, p. 18 de 20/12/2005, 2005.

DELOUCHE, J. C.; BASKIN, C. C. Accelerated agingtechniques for predicting the relative storability of seed lots. Seed Science and Technology, Zurich, v. 1, n. 2, p. 427-452, 1973.

DIAS, D. C. F. S.; MARCOS FILHO, F. Testes de vigor baseados na permeabilidade das membranas celulares: II. Lixiviação de potássio. Informativo ABRATES, Londrina, v. 5, n. 1, p. 37-41, 1995.

DIAS, D. C. F. S.; MARCOS FILHO, J. Testes de condutividade elétrica para avaliação do vigor de sementes de soja. Scientia Agricola, Piracicaba, v. 53, n. 1, p. 31-42, 1996.

DIAS, D. C. F. S.; VIEIRA, A. N.; BHÉRING, M. C. Condutividade elétrica e lixiviação de potássio para avaliação do vigor de sementes de hortaliças: feijão-devagem e quiabo. Revista Brasileira de Sementes, Brasília, v. 20, n. 2, p. 408-413, 1998. 
DUTRA, A. S.; VIEIRA, R. D. Teste de condutividade elétrica para a avaliação do vigor de sementes de abobrinha. Revista Brasileira de Sementes, Pelotas, v. 28, n. 2, p. 117-122, 2006.

FORNAZIERI JÚNIOR, A. Mamona: uma rica fonte de óleo e de divisas. São Paulo: Ícone, 1986.

GASPAR, C. M.; NAKAGAWA, J. Teste de condutividade elétrica em função do período e da temperatura de embebição para sementes de milheto. Revista Brasileira de Sementes, Brasília, v. 24, n. 2, p. 82-89, 2002.

GOMES, F. P. Curso de estatística experimental. 14. ed. Piracicaba: Escola Superior de Agricultura Luiz de Queiroz, 2000. 477 p.

HAMPTON, J. G.; JOHNSTONE, K. A.; EUAUMPON, $\mathrm{V}$. Bulk conductivity test variables for mungbean, soybean and French bean seed lots. Seed Science and Technology, Zurich, v. 20, n. 3, p. 677-686, 1992.

HAMPTON, J. G.; TREKONY, D. M. Handbook of vigour test methods. Zürich: ISTA, 1995. 117 p.

LOEFFLER, T. M.; TEKRONY, D. M.; EGLI, D. B. The bulk conductivity test as an indicator of soybean seed quality. Journal of Seed Technology, Lansing, v. 12, n. 1, p. 37-53, 1988.

MAGUIRE, J. D. Speed of germination: aid in selection and evaluation for seedling emergence and vigor. Crop Science, Madison, v. 2, n. 2, p. 176-7, 1962.

MARCOS FILHO, J. Testes de vigor: importância e utilização. In: KRZYZANOWSKI, F. C.; VIEIRA, R. D.; FRANÇA NETO, J. B. (Ed.). Vigor de sementes: conceitos e testes. Londrina: ABRATES, 1999. Cap. 1, p. 1-21.

MARCOS FILHO, J.;SILVA, W. R.; NOVEMBRE, A. D. C.; CHAMMA, H. M. C. P. Estudos comparativos de métodos para avaliação da qualidade fisiológica de sementes de soja, com ênfase ao teste de condutividade elétrica. Pesquisa Agropecuária Brasileira, Brasília, v. 25, n. 12, p. 1805-1815, 1990.

MARTINS, C. C.; MARTINELLI-SENEME, A.; CASTRO, M. M.; NAKAGAWA, J.; CAVARIANI, C. Comparação entre métodos para a avaliação do vigor de lotes de sementes de couve-brócolos (Brassica oleracea 1. var. italica plenk). Revista Brasileira de Sementes, Brasília, v. 24, n. 2, p. 96-101, 2002.

MENEZES, N. L.; PASINATTO, P. R. Protocolo do teste de condutividade elétrica para avaliação do vigor de sementes de azevém, aveia preta e milheto. Informativo ABRATES, v. 5, n. 2, p. 123-123. 1995.
POWELL, A. A. Cell membranes and seed leachate conductivity in relation to the quality of seed for sowing. Journal of Seed Technology, Lincoln, v. 10, n. 2, p. 81100,1986

RIBEIRO, D. M. C. A.; CARVALHO, M. L. M.; SALGADO, K. C. C. Avaliação da qualidade fisiológica de sementes de milho através do teste de condutividade elétrica (bulk). In: CONGRESSO BRASILEIRO DE SEMENTES, 10., 1997, Foz do Iguaçu. Anais... Foz do Iguaçu, 1997. p. 187.

RODO, A. B.; TILLMANN, M. A. A.; VILLELA, F. A.; SAMPAIO, N. V. Teste de condutividade elétrica em sementes de tomate. Revista Brasileira de Sementes, Brasília, v. 20, n. 1, p. 29-38, 1998.

SÁ, M. E. Condutividade elétrica em sementes de tomate (Lycopersicon lycopersicum L.). Scientia Agricola, Piracicaba, v. 56, n. 1, p. 13-19, 1999.

SOUZA, S. A.; CAVARIANI, C.; NAKAGAWA, J.; MARTINS, C. C. Accelerated aging test in castor seeds (Ricinus communis L.). In: INTERNATIONAL SEED CONGRESS, 28., 2007, Foz do Iguaçu. Resumos... Foz do Iguaçu, 2007. p. 112.

TORRES, S. B.; MARCOS FILHO, J. Teste de envelhecimento acelerado em sementes de maxixe (Cucumis anguria L.). Revista Brasileira de Sementes, Brasília, v. 23, n. 2, p. 108-112, 2001.

VANZOLINI, S. Teste de condutividade elétrica em sementes de amendoim (Arachis hypogea L.). 1998. Dissertação (Mestrado em Agronomia) - Universidade Estadual Paulista, Depto Agricultura e Melhoramento Vegetal, 1998.

VIEIRA, R. D.; PANOBIANCO, M.; LEMOS, L. B.; FORNASIERI FILHO, D. Efeito de genótipos de feijão e de soja sobre os resultados da condutividade elétrica de sementes. Revista Brasileira de Sementes, Brasília, v. 18, n. 2, p. 220-224, 1996.

VIEIRA, R. D.; PENARIOL, A. L.; PERECIN, D.; PANOBIANCO, M. Condutividade elétrica e teor de água inicial das sementes de soja. Pesquisa Agropecuária Brasileira, Brasília, v. 37, n. 9, p. 1333-1338, 2002.

VIEIRA, R. D.; KRZYZANOWSKI, F.C. Teste de condutividade elétrica. In: KRZYZANOWSKY, F. C.; VIEIRA, R. D.; FRANÇA-NETO, J. B. Vigor de sementes: conceitos e testes. Londrina: ABRATES, 1999. p.4/1-4/26.

WANG, Y. R.; HAMPTON, J. G.; HILL, M. J. Red clover vigour testing: effects of three test variables. Seed Science and Technology, Zurich, v. 22, n. 1, p. 99-105, 1994. 